\title{
Combination of Shengji ointment and bromelain in the treatment of exposed tendons in diabetic foot ulcers: Study protocol for a non-blind, randomized, positive control clinical trial
}

\section{yang zhao}

China Academy of Chinese Medical Sciences Xiyuan Hospital

rui gao ( $\nabla$ ruigao@126.com )

China Academy of Chinese Medical Sciences Xiyuan Hospital https://orcid.org/0000-0003-2455-7615

\section{Xinyue Dai}

China Academy of Chinese Medical Sciences

\section{Xu Sun}

The second affiliated hospital of tianjin university of traditional chinese medicine

\section{Zhaohui Zhang}

The second affiliated hospital of tianjin university of traditional chinese medicine

Hongyang Gao

China Academy of Chinese Medical Sciences Xiyuan Hospital

\section{Research Article}

Keywords: Administration, topical, Bromelain, Diabetic foot, Herbal medicine, Medicine, Chinese Traditional, Ointment, Tendon injuries

Posted Date: September 21st, 2021

DOI: https://doi.org/10.21203/rs.3.rs-442956/v1

License: (c) (i) This work is licensed under a Creative Commons Attribution 4.0 International License. Read Full License 
1 Combination of Shengji ointment and bromelain in the treatment of

2 exposed tendons in diabetic foot ulcers: Study protocol for a non-blind,

3 randomized, positive control clinical trial

4 Protocol contributors: Yang Zhao ${ }^{1,4,5}$, Xinyue Dai ${ }^{2}, \mathrm{Xu} \mathrm{Sun}^{3}$, Zhaohui Zhang ${ }^{3}$,

5 Hongyang $\mathrm{Gao}^{1}$, Rui Gao ${ }^{1,4,5 *}$

$6 \quad{ }^{1}$ Xiyuan Hospital China Academy of Chinese Medical Sciences, Beijing, China.

$7 \quad{ }^{2}$ China Academy of Chinese Medical Sciences, Beijing, China.

$8{ }^{3}$ The Second Affiliated Hospital of Tianjin University of Traditional Chinese

9 Medicine, Tianjin, China.

$10{ }^{4}$ NMPA Key Laboratory for Clinical Research and Evaluation of Traditional

11 Chinese Medicine, Beijing, China.

$12{ }^{5}$ National Clinical Research Center for Chinese Medicine Cardiology, Beijing,

13 China.

14 *Corresponding author: Rui Gao, ruigao@ 126.com. 


\section{Abstract}

\section{Background:}

21 Diabetic foot ulcers often affect tendon tissue. Consequently, the infection may

22 spread proximally along the tendon, leading to amputation or even the death of

23 patients. Exposed, degenerated, and necrotic tendons are key factors affecting

24 the healing of diabetic foot ulcers. The effective treatment of the tendon

25 involvement may positively affect the prognosis. In clinical practice, treatment

26 with Shengji ointment and bromelain induces islands of granulation tissue on

27 the denatured tendon surface, which gradually grows and merges. Ideally, the

28 exposed tendon is covered entirely by granulation tissue.

29 This trial aims to assess the effect of a combined treatment regime of Shengji

30 ointment, which has been shown to regenerate muscle tissue and pineapple

31 protease in preventing the loss of function and amputation caused by tendon

32 necrosis. This trial will provide high-quality evidence for the effectiveness of

33 this combination in healing diabetic ulcers with tendon necrosis.

\section{Methods:}

35 The sample size will be 180 patients who will be randomly assigned 1:1 to a

36 treatment group (90 patients) using Shengji ointment combined with bromelain

37 and a control group (90 patients) using hydrocolloid dressing. Both groups will

38 continue their conventional treatments, such as blood glucose and blood 
39 pressure medication, lipid regulation, antiplatelets, and others. The primary

40 outcome will be the wound coverage with granulation tissue. Secondary

41 outcomes will be the wound healing rate, amputation extent (where needed),

42 time to granulation, and the Maryland Foot Score. Other efficacy outcomes will

43 be the time to debridement of necrotic tendon tissue and granulation tissue score.

\section{Discussion:}

45 This study will treat patients with diabetic foot ulcers with exposed, degenerated,

46 and necrotic tendons with Shengji ointment and bromelain. The trial aims to

47 promote regeneration and healing, to preserve the limb and its function, and to

48 develop a comprehensive and effective protocol that can be applied to promote

49 the healing of exposed tendons in diabetic foot wounds.

50 Trial registration: ChiCTR2000039327; date of registration: 2020-10-23.

51 http://www.chictr.org.cn/com/25/showproj.aspx?proj=62692

53 Keywords: Administration, topical; Bromelain; Diabetic foot; Herbal medicine;

54 Medicine, Chinese Traditional; Ointment; Tendon injuries 
58 Administrative information

59 Note: the numbers in curly brackets in this protocol refer to SPIRIT checklist

60 item numbers. The order of the items has been modified to group similar items

61 (see http://www.equator-network.org/reporting-guidelines/spirit-2013-

62 statement-defining-standard-protocol-items-for-clinical-trials/).

63

\begin{tabular}{|c|c|}
\hline Title $\{1\}$ & $\begin{array}{l}\text { Combination of Shengji ointment and bromelain for } \\
\text { the treatment of exposed tendons in diabetic foot } \\
\text { ulcers: Study protocol for a non-blind, randomized, } \\
\text { positive control clinical trial }\end{array}$ \\
\hline $\begin{array}{l}\text { Trial registration }\{2 \mathrm{a} \text { and } \\
2 \mathrm{~b}\} \text {. }\end{array}$ & ChiCTR2000039327 \\
\hline Protocol version $\{3\}$ & V3.0 \\
\hline Funding $\{4\}$ & $\begin{array}{l}\text { National key research and development plan funding } \\
\text { (2019YFC1709300) }\end{array}$ \\
\hline Author details $\{5 \mathrm{a}\}$ & $\begin{array}{l}\text { Yang Zhao }{ }^{145}, \text { Xinyue } \text { Dai }^{2}, \text { Xu Sun }{ }^{3} \text {, Zhaohui Zhang } \\
\text {, } \\
\text { Hongyang Gao }{ }^{145} \text {, Rui Gao }{ }^{145} \\
{ }^{1} \text { Xiyuan Hospital China Academy of Chinese Medical }\end{array}$ \\
\hline
\end{tabular}




\begin{tabular}{|c|c|}
\hline & $\begin{array}{l}\text { Sciences } \\
{ }^{2} \text { China Academy of Chinese Medical Sciences } \\
{ }^{3} \text { Second Affiliated Hospital of Tianjin University of } \\
\text { TCM } \\
{ }^{4} \text { NMPA Key Laboratory for Clinical Research and } \\
\text { Evaluation of Traditional Chinese Medicine } \\
{ }^{5} \text { National Clinical Research Center for Chinese } \\
\text { Medicine Cardiology }\end{array}$ \\
\hline $\begin{array}{l}\text { Name and contact } \\
\text { information for the trial } \\
\text { sponsor }\{5 b\}\end{array}$ & $\begin{array}{l}\text { Rui Gao, Xiyuan Hospital China Academy of Chinese } \\
\text { Medical Sciences, Haidian District, Beijing, China. }\end{array}$ \\
\hline Role of sponsor $\{5 c\}$ & $\begin{array}{l}\text { Study design, writing of the report, and the decision } \\
\text { for publication. }\end{array}$ \\
\hline
\end{tabular}

66 Background and rationale $\{\mathbf{6 a}\}$

67 A diabetic foot is defined as the destruction of skin and deep tissues distal from

68 the ankle joint in patients with diabetes, often combined with infection and/or

69 peripheral arterial occlusive disease of varying degree in the lower limbs and, in

70 severe cases, involving muscle and bone tissue [1]. Diabetic foot is one of the 
most common and serious chronic complications of diabetes and a frequent

cause of disability and death [2]. It is estimated that a limb is amputated every

$20 \mathrm{~s}$ in patients with diabetes worldwide [3]. Diabetic foot ulcers often extend

deep into tissue and the underlying tendons [4], and the infection can spread proximally along the tendon, eventually leading to amputation or death.

Exposed, degenerated, and necrotic tendons impair the healing of diabetic foot ulcers, and the effective treatment of these tendon injuries improves the prognosis of wound healing $[5,6]$.

The International Diabetic Foot Working Group guidelines emphasize that the management of diabetic foot ulcers requires a multidisciplinary and comprehensive approach [7]. In clinical Traditional Chinese Medicine practice, denatured but still vital tendon surfaces are covered with patches of new granulation tissue that gradually grow and eventually merge with each other when treating diabetic ulcers with a combination of Shengji ointment and bromelain.

In Traditional Chinese Medicine (TCM), treatment according to the method of Hua Fu Sheng Ji entails bromelain, which targets liquefied necrotic tendon and fascia, removes necrotic tissue, and prevents further putrefaction, thereby providing a clean wound bed that promotes healing in diabetic ulcers [8]. The combination of bromelain with Shengji ointment uses the synergistic effects of the substances and promotes the in situ regeneration of granulation tissue and 
92 epithelial tissue. It has been shown that the content of total protein, total amino

93 acid, and lysozyme per unit of wound exudate increases significantly over

94 different periods of time under this treatment, providing the nutrients for wound

95 healing [9]. This may result in a reversal of the denaturation of the tendon tissue

96 and stimulates the growth of granulation tissue that starts to cover tendon tissue,

97 which may allow to retain parts of the tendon and to preserve foot function to a 98 certain extent.

99 Previous animal studies [9] have shown that the application of bromelain

100 combined with Shengji ointment can target and remove necrotic tendon tissue,

101 promote tissue regeneration, and realize tissue repair in situ. The healing rate

102 was $60 \%$, and the efficacy rate was $94.3 \%$. By changing the $\mathrm{pH}$-value and

103 lysozyme content of the wound, the method may liquefy the necrotic tendon and

104 fascia, control infection, and stop decay. The TCM ointment Shengji mainly

105 focuses on the growth of muscle and skin by providing amino acids, proteins,

106 and other substances needed for wound repair and stimulating epidermal growth.

107 The combination of Shengji with bromelain can accelerate tissue growth and

108 promote wound healing. Our preliminary research [National Natural Science

109 Foundation Project (30873270): Mechanism Research on the Effect of

110 Decomposing Rot and Promoting Muscle Growth on Diabetic Foot Tendon

111 Necrosis] has found that some degenerated tendon tissue recovered after

112 treatment, and granulation tissue grew to cover the tendon tissue, retaining parts 
113 of the tendons and preserving foot function to a certain extent, which we called

114 the "Jin Zhi Xue Hua" phenomenon [10]. Later, based on further research

115 [National Natural Science Fund Project (81573972), we found that the creation

116 of a steady-state microenvironment promoted the perfusion of tendons during

117 the healing of diabetic foot ulcers] and concluded that "the method of

118 Hua Fu Sheng Ji can promote a stable microenvironment in diabetic foot ulcers."

119 It is of great significance to explore the underlying mechanisms to improve

120 diabetic foot ulcer repair and to enrich the theory of topical treatment in TCM.

121 This clinical trial will follow a multicenter, randomized, positive control design

122 aimed at assessing the combination treatment's efficacy in promoting tendon

123 healing and preserving both the limb and its function in patients with Wagner

124 grade 3-4 diabetic foot ulcers and tendon exposure.

125 The trial aimed to a) investigate the effect of Shengji ointment and pineapple

126 protease, used for muscle regeneration and healing, as therapeutic drugs in

127 preventing amputation necessitated by tendon degeneration and necrosis,

128 thereby preserve limb function, and to b) develop high-quality evidence and a

129 comprehensively effective plan for promoting the healing of exposed tendons in 130 diabetic foot ulcers. 
133 The objectives of this trial are as follows:

134 1. Create high-quality evidence on the effects of the treatment of diabetic ulcers

135 with a combination of Shengji ointment;

136 2. Evaluate the efficacy and safety of the combination treatment;

137 3. Develop a generalizable TCM treatment plan for diabetic foot ulcers with

138 tendon exposure.

140 Trial design $\{8\}$

141 This study was a multicenter, non-blind, randomized, positive control trial.

143 Methods: Participants, interventions, and outcomes

144 Study setting $\{9\}$

145 The diagnostic criteria for diabetic foot refer to

146 (1) the Technical Guiding Principles for Clinical Research of New

147 Traditional Chinese Medicine for Diabetic Foot (Draft for Soliciting

148 Opinions [1]) and the Guidelines for Diagnosis and Treatment of Diabetic 

the State Drug Administration; (2) Diabetic foot grading standard: Wagner grading [16].

154 Inclusion criteria

155 Patients who meet the following criteria will be included:

156 (1) Diagnostic criteria for diabetic foot disease and Wagner grade 3-4 ulcers

157 with exposed tendon tissue;

$158 \quad$ (2) Age between 18 and 85 years;

$159 \quad$ (3) Fasting blood glucose $\leq 10 \mathrm{mmol} / \mathrm{L}$;

160 (4) Targeted ulcer debridement area between 1 and $20 \mathrm{~cm}^{2}$ (for patients with 161 multiple lesions, the largest ulcer will be the target lesion);

162 (5) An ankle-brachial index $\geq 0.5$ on the side of the limb with the ulcer;

163 (6) The ulcer has blood, pus, or sticky secretion;

164 (7) Voluntary participation and signing of an informed consent form.

165 Exclusion criteria

166 Patients who meet any of the following criteria will be excluded: 
(1) Skin ulcer caused by electrical, chemical, or radiation injury, tumors, varicose veins, or other reasons; or malignant lesions within the ulcer;

(2) Severe clinical infection indicated by cellulitis, fever, elevated white blood cell count, bacterial culture, or increased (high-sensitivity) Creactive protein levels;

(3) Severe uncontrollable hypertension with systolic blood pressure $\geq 160$ $\mathrm{mmHg}$ or diastolic blood pressure $\geq 110 \mathrm{mmHg}$;

(4) Serum albumin levels < $28 \mathrm{~g} / \mathrm{L}$;

(5) Hemoglobin < $90 \mathrm{~g} / \mathrm{L}$;

(6) Platelet count $<50 \times 10^{9} / \mathrm{L}$;

(7) Severe heart, liver, or kidney injury, in case of medical treatment that may seriously affect the safety and treatment;

(8) Pregnancy, family planning, or breastfeeding women;

(9) Cognitive dysfunction preventing fully informed consent;

186

(10) Allergic disposition or allergic to the ingredients of the treatment under investigation and reference drugs;

(11) Participation in other clinical trials during the past one month;

(12) In the judgment of the researcher, inability to complete the trial or comply with its requirements. 
188 Before a patient is enrolled in this trial, the researcher is responsible for

189 explaining the purpose, nature, procedure, possible benefits, and risks of their

190 participation to them or their designated representative in written form. Patients

191 shall be informed that they have the right to withdraw from the study at any

192 time. A written informed consent form must be given to each patient before

193 enrollment such that they can confirm that they understand and agree with their

194 participation. Informed consent forms must be voluntarily signed before the

195 patients are enrolled in the trial. The informed consent form will be kept as one

196 of the original materials for the trial.

198 Interventions

199 Explanation for the choice of comparators $\{6 b\}$

200 As a commonly used dressing for diabetic foot wounds, the hydrocolloid

201 dressings are safe, reliable, effective, and superior in the treatment of diabetic

202 foot ulcers and other chronic difficult-to-treat wounds. The hydrocolloid

203 dressing was determined to be the best comparator as a positive control. 
206 Both the intervention and control groups will receive routine medical and

207 surgical treatment (blood sugar control, blood pressure reduction, lipid

208 regulation, antiplatelet medication, debridement, and others).

209 In the intervention group, bromelain powder will be applied to the exposed

210 tendon and necrotic tissue, and the wound will be covered with Shengji

211 ointment. In the control group, a hydrocolloid dressing will be used to cover the

212 wound.

213 Treatment 1: Shengji ointment

214 Size: $30 \mathrm{~g} / \mathrm{box}$

215 Formulation: Ointment

$216 \quad$ Usage and dosage: For external use. The ointment will be spread on

217 skimmed cotton and applied to the affected area.

$218 \quad$ Route of administration: Topical

219 Frequency of administration: Once every $24 \mathrm{~h}$

$220 \quad$ Treatment course: Four weeks

221 Manufacturer: Tianjin Darentang Jingwanhong Pharmaceutical Co., Ltd,

222 Tianjin, Tianjin, China 
223 Treatment 2: Pineapple protease (bromelain) with necrotic tissue.

231 Manufacturer: Shantou Olive Pharmaceutical Co., Ltd., Shantou City,

232 Guangdong, China

233 Active control: Comfeel® Plus wound dressing

$234 \quad$ Size: $25 \mathrm{~g} /$ piece

235 Formulation: Hydrocolloid dressing

236 Usage and dosage: Apply Comfeel® dressing to the ulcer. Ensure the

237 dressing height is at the level of the surrounding skin. Then, apply a layer of 238 dressing over the ulcer and surrounding area. 
244 Criteria for discontinuing or modifying allocated interventions $\{11 \mathrm{~b}\}$

245 In enrolled patients who withdraw from the trial without completing the clinical

246 protocol for any reason during the trial, two conditions will be distinguished:

247 researcher-determined discontinuation and voluntary withdrawal.

248 I. Termination of a patient's participation based on the researcher's decision

249 The researchers may decide to withdraw enrolled subjects from the study when

250 they establish that they are not suitable to continue the study for any of the

251 following reasons:

252 (1) During the trial, clinical endpoints (such as the need for surgery) are 253 reached.

254 (2) The patient undergoes surgical treatment other than debridement of the ulcer site. 
(3) During the clinical trial, patients experience complications or physiological changes that make it inappropriate for them to continue to receive treatment within the trial.

(4) Use of other prohibited treatments or drugs that affect the assessment of the efficacy and safety of the intervention.

(5) If serious adverse events occur during the trial, the researcher may decide whether to suspend the trial.

(6) Poor compliance of the patients that results in the failure of $80 \%$ of the prescribed dosage of topical drugs (except cured patients) or the failure of

266 Subjects who will be withdrawn from the trial will undergo a final visit to

267 evaluate each efficacy indicator and to complete a safety assessment.

2. Patients who voluntarily withdraw from the trial

269 Every patient has the right to withdraw from the trial in accordance with the 270 Good Clinical Practice (GCP) and informed consent. "Withdrawal" also refers

271 to the loss of a patient after he/she no longer receives the treatment and

272 assessment of the intervention, although he/she does not explicitly propose

273 withdrawal from the study. 
(1) If the intervention or control is found to be ineffective, the patient may be unwilling to continue the trial. If the patient proposes to withdraw from the trial to the researcher before returning to the routine treatment.

(2) Patients who, due to various other reasons, are unwilling or unable to continue the clinical trial and terminate the trial by offering to withdraw from the trial to the researcher.

(3) Although the patients do not explicitly propose withdrawing from the study, they are no longer followed up and are thus lost.

282 Researchers will aim to learn as much as possible about the reasons for patients'

283 withdrawal and record them. For example, the perceived curative effect may not

284 be good; patients may not tolerate some adverse reactions; patients may be

285 unable to continue the clinical trial for various reasons, e.g., economic factors;

286 or loss of follow-up without explanation.

287 II. Suspension/termination criteria for the whole trial:

288 Suspension/termination of the trial means that the entire clinical trial is not

289 completed in accordance with the protocol. The purpose of a trial

290 suspension/termination is to protect patients' rights and interests, to ensure the

291 quality of the trial, and to avoid unnecessary economic losses.

292 This trial may be discontinued for the following reasons: 
(1) When serious safety problems occur during the trial and the researcher believes that the safety of the patients may be compromised, or when the treatment is found to be too poor or ineffective during the trial to have a clinical value.

(2) It is difficult to evaluate the efficacy and/or safety of the treatment because major errors are discovered in the trial protocol or significant deviations in its implementation occur during the trial.

(3) The researcher proposes suspension (for example, funding or management reasons, or others).

The researcher will notify the patient, organization responsible for the trial, and Ethics Committee and explain the reasons for discontinuing the trial. The responsible research unit shall notify the investigator, Ethics Committee, and Ministry of Science and Technology before suspending the clinical trial and state the reasons.

\section{Relevant concomitant care permitted or prohibited during the trial $\{11 d\}$}

No additional Chinese or Western drugs related to the treatment of the ulcer (vasodilators, such as lipid microspheres prostaglandin injection, beraprost sodium, cilostazol, sagresol hydrochloride, nefuram, butalbital, and hexaketone cocaine, among others; antiplatelet drugs, such as aspirin and clopidogrel; anti- 
313 coagulant drugs, such as unfractionated heparin or low-molecular-weight

314 heparin, and oral anti-coagulants; or TCM or proprietary Chinese medicine,

315 topical antibiotics, trimethoprim/sulfamethoxazole, and others, which have the

316 effect of muscle growth and act as astringents in the treatment of sores) should

317 be used during the trial. The same applies to biological treatments (such as stem

318 cell therapy, topical autologous platelet-rich plasma, maggot therapy, cell

319 growth factors, chymotrypsin, and TCM or Chinese herbal tonics with similar

320 functions as the trial treatment).

321 Drugs that are required to treat comorbidities may be continued. These drugs

322 must be recorded in detail on the case report form, including the name of the

323 drug, dosage, frequency, and duration of use.

324 Glucose-lowering drugs (oral antidiabetics and insulin) will be selected

325 according to the patient's blood glucose level at the time of enrollment and

326 treatment regimen. Except for uncontrolled hyperglycemia, the type of glucose-

327 lowering drug or insulin regimens of patients will remain unchanged, although

328 the dosage will be adjusted according to blood glucose levels. Blood glucose

329 levels and glucose-lowering drug doses will be recorded in a timely and detailed

330 manner on the case report form. Patients whose blood glucose levels cannot be

331 normalized within a week will be discontinued from the trial. 
335 The wound coverage with granulation tissue will be the primary outcome. The 336 development of granulation tissue will be assessed as follows:

337 Wound coverage rate $=$ The wound area covered by granulation tissue

$338\left(\mathrm{~mm}^{2}\right) /$ Whole wound area $\left(\mathrm{mm}^{2}\right) \times 100 \%$

339 We will use three-dimensional scanning to obtain the wound surface topography

340 and the inSight ${ }^{\circledR}$ platform (eKare, Inc., Fairfax, VA, USA) to identify the

341 different tissue types and to measure the areas covered by them.

343 Secondary outcomes

344 As secondary outcomes, we will determine the wound healing rate, amputation

345 rate and extent, granulation time, and Maryland Foot Score.

346 (1) Wound healing rate $=($ Original wound area - unhealed wound area $) /$ Original

347 wound area $\times 100 \%$.

348 (2) Amputation extent: A extensive amputation refers to an amputation above

349 the ankle, while a limited amputation refers to an amputation below the ankle.

350 The amputation level will be identified in a multidisciplinary team of vascular 
351 surgeons and orthopedic foot and diabetes specialists according to the results of

352 the lower limb angiography.

353 (3) Granulation time (days): The period of time within which new granulation

354 tissue appears within the wound.

355 (4) The Maryland Foot Score will be determined to evaluate foot function,

356 including the presence or absence of pain. The maximum score is 100 points, >

35789 points indicate excellent function, $75-89$ points good function, 50-74

358 average, and $<50$ points poor function.

360 Other indicators of treatment efficacy

361 We will also record the following parameters:

362 Clearance time (days): The period from enrollment (day 0) to the complete

363 clearance of degenerated and necrotic tendon tissue.

364 Granulation tissue score: Evaluation of scores at enrollment (day 0), visit 2

365 (week 2), and at the end of the trial (week 4). 
368 The time schedule of the enrollment, interventions, assessments, and visits for

369 the participants in this trial is shown in Table 1.

370 Sample size $\{14\}$

371 The sample size was calculated using Pass 11.0 software (NCSS Statistical

372 software https://www.ncss.com/software/pass). Based on a preliminary review

373 of the literature and the clinical practice and experience of the Second Affiliated

374 Hospital of Tianjin University of Traditional Chinese Medicine in the treatment

375 of diabetic foot ulcers, the control group is expected to have an effectiveness of

$37650 \%$, and the treatment group of $71 \%$. Postulating a patient ratio of 1:1 between

377 groups and an alpha of 0.05 on both sides, 72 patients would be required in each

378 group. Assuming a 20\% loss to follow-up, we calculated a number of 90

379 patients in each group and a total of 180 patients as the target sample size.

381 Recruitment $\{15\}$

382 The patients will be followed up at four hospitals; the Second Affiliated

383 Hospital of Tianjin University of Traditional Chinese Medicine (80 patients),

384 Affiliated Hospital of Liaoning University of Traditional Chinese Medicine (40

385 patients), Affiliated Hospital of Shanxi University of Traditional Chinese

386 Medicine (40 patients), and Tianjin Binhai New Area Hospital of Traditional

387 Chinese Medicine (20 patients). 
388 Assignment of interventions: random allocation.

389

390 Sequence generation $\{16 a\}$

391 Using a central random system and minimization algorithm, the allocation

392 probability of the target group will be calculated for each enrolled patient for

393 them to be assigned to the most suitable treatment group and ensuring a balance

394 of control factors between the groups. The controlling factors are 1) ulcer

395 location (plantar, dorsum of foot, or toe), and 2) ABI evaluation index (> 0.7,

396 and $\leqslant 0.7)$.

397

398 Concealment mechanism $\{16 b\}$

399 None.

400

401 Data collection and management

402 Data management $\{19\}$

403 1. Research medical records 
404 Since most outpatient medical records at China's hospitals are kept by the

405 patients themselves, the case report form will be designed specifically for this

406 trial to preserve first-hand information. The research medical records are the

407 original documents of the trial patients, which are properly kept by each trial

408 center.

409 2. Electronic data management

410 The electronic case report form is created using the clinical trial data

411 management system (eCDMS3.0) at Xiyuan Hospital developed by the Chinese

412 Academy of Traditional Chinese Medicine, and the data will be collected and

413 managed online.

414 (1) Full analysis set: This refers to patients receiving the allocated treatment at

415 least once and having data on at least one post-treatment primary outcome

416 measurement. For patient data that fail to observe the whole treatment process,

417 the data from the last observation will be carried forward to the final last

418 observation carried forward.

419 (2) Per protocol set: Good compliance $(80 \% \leq$ treatment compliance $\leq 120 \%)$,

420 not taking prohibited medications during the study, and no serious violation of

421 the trial protocol. 
422 (3) Safety set: All patients who are randomized and use the trial treatment at 423 least once.

424

425 Statistical methods for primary and secondary outcomes $\{\mathbf{2 0 a}\}$

426 1. Statistical description

427 (1) Detailed description of cases of withdrawal and censoring, including the 428 time and reason for censoring.

429 (2) Descriptive statistics: Based on their distribution, data will be presented as

430 either the mean and standard deviation, or the median with maximum, minimum,

431 and confidence interval, or the number and frequency (\%), among others.

432 2. Statistical inference method

433 (1) Measurement data: The paired t-test, analysis of variance, the rank-sum test, 434 signed rank-sum test, and the median test will be used.

435 (2) Counting data: The chi-square test, Fisher exact test, and others will be used;

436 grade data will be analyzed using the ridit test and the Cochran-Mantel437 Haenszel test.

438 (3) Primary outcome analysis: Per protocol set analysis and full analysis set 439 analysis will be performed. The Cochran-Mantel-Haenszel test will be used for 
440 multicenter counts and analysis of covariance for measures. For the

441 confounding factors that are difficult to control or are uncontrolled before

442 allocation, the least-squares means of the analysis of covariance and their $95 \%$

443 confidence limit or logistic regression will be used as covariates to determine

444 the between-group efficacy.

445 (4) Two-sided difference tests will be used to assess statistical differences, and a

446 difference with a $p$-value of $\leq 0.05$ will be considered to be statistically

447 significant.

448

449 Oversight and monitoring

450 Quality control

451 Researcher authorization: All researchers are allowed to enter the trial and to

452 conduct trial operations within the scope of authorization only after receiving

453 training and authorization from the Principal Investigator.

454 Clinical monitoring: This clinical trial will be supervised by a clinical research 455 auditor, who instructs the researchers to conduct the clinical trial in accordance

456 with the trial protocol and GCP.

457

458 Quality assurance 
459 Researcher training: Before the start of the trial, the principal investigation unit 460 is required to train all researchers involved in the trial and others associated 461 with the trial on the GCP and the trial protocol.

462

463 Standard Operating Procedures

464 All researchers must strictly follow the standard operating procedures provided 465 for this trial.

466

467 Auditing

468 The sponsor is responsible for auditing the trial and providing proof of audit to 469 the Principal Investigator.

470

471 Adverse event reporting and harms $\{22\}$

472 The adverse events, adverse reactions, and serious adverse events in this study

473 will be recorded. The safety assessment will include the recording and analysis 474 of

475 1. All adverse events (including symptoms, signs, etc.);

476 2. Liver and kidney function, hematuria; stool routine, and

$477 \quad$ electrocardiogram;

478 3. Pregnancy tests in women as indicated. 
481 The exposure, degeneration, and necrosis of tendons are key factors affecting 482 the healing of diabetic foot ulcers, and the successful treatment of the tendon 483 injury affects prognosis. Clinical practice has shown that Shengji ointment 484 combined with bromelain treatment can induce granulation islands to form on 485 the denervated but not yet inactive tendons. These islands gradually grow 486 together, eventually covering the previously exposed tendon with granulation 487 tissue to heal the wound.

488 This multicenter, randomized, positive control trial will evaluate the clinical 489 effects, validate the efficacy and safety of this combined treatment with the aim 490 of developing a generalizable TCM treatment plan for diabetic foot ulcers with 491 exposed tendons, and provide high-quality evidence for its clinical applications.

\section{Trial status}

494 Protocol version number and date: Version 3.0, 23 Aug 2020.

495 The study was registered at the Chinese Clinical Trials Registry on 23 Oct 2020, 496 registration number: ChiCTR2000039327. Recruitment was started on 1 Dec 4972020 and is expected to end in December 2022. 498 499 Abbreviations 
500 GCP Good Clinical Practice

501 TCM Traditional Chinese Medicine

502

503 Declarations

504 Ethics approval and consent to participate $\{24\}$

505 Institutional ethics approval for the trial was obtained from the Ethics

506 Committee of the Second Affiliated Hospital of Tianjin University of Chinese

507 Medicine. Informed consent will be obtained from all participants. Study

508 investigators and treating physicians will serve as guarantors for keeping the

509 data confidential for all participants in each trial, following the established

510 privacy rules of clinical practice. Patients will be asked for their permission for

511 the data analysis conducted anonymously for research purposes. All study

512 investigators will have access to the final trial data set. Trial results will be

513 disseminated via publication and the study participants will be informed directly.

514 Ethical approval document is attached as additional file 5.

515

516 Consent for publication $\{32\}$

517 Not applicable.

519 Availability of data and material \{29\}

520 Not applicable. 
523 No competing interests.

525 Funding $\{4\}$

526 National key research and development plan funding support. Copy of the

527 original funding documentation has been provided, see additional file 6 .

529 Authors' contributions $\{31 b\}$

$530 \mathrm{RG}$ and $\mathrm{ZZ}$ conceived the research and developed the first version of the

531 protocol. XS revised the protocol, YZ, HG and XD drafted the manuscript, and 532 all other authors contributed to the editing of the final manuscript and approved 533 the final version.

535 Acknowledgments

536 The authors acknowledge the contribution of the staff of the four hospitals; the

537 Second Affiliated Hospital of Tianjin University of Traditional Chinese

538 Medicine, the Affiliated Hospital of Liaoning University of Traditional Chinese

539 Medicine, the Affiliated Hospital of Shanxi University of Traditional Chinese

540 Medicine, and the Tianjin Binhai New Area Hospital of Traditional Chinese

541 Medicine. We thank Editage (www.editage.cn) for the English language editing. 


\section{References}

545 1. Chinese guidelines for the prevention and treatment of diabetic foot (2019

546 edition) (I) [J]. Chinese Journal of Diabetes. 2019;2:92-108.

547 2. China Healthcare International Exchange Promotion Association Diabetic

548 Podiatry Branch and International Vascular Federation China Chapter Diabetic

549 Podiatry Expert Committee, Guidelines for the diagnosis and treatment of

550 diabetic foot in China. Chinese Journal Clinical. Physician's, 2020;48:19-27.

551 3. Ibrahim A. IDF clinical practice recommendation on the diabetic foot: a

552 guide for healthcare professionals. Diabetes Res Clin Pract. 2017;127:285-7.

553 4. Qiang X, Chaohui Z, Chaojun Z. A preliminary study on promoting the

554 "blood of tendons" of diabetic foot with the theory of giving away evil. Chin J

555 Basic Med Trad Chin Med. 2015;21:22-3.

556 5. Qiang X, Chaohui Z, Jing M, Chaojun Z, Xianzhou L. Preliminary study

557 on the treatment of diabetic foot by the method of blood and decay regeneration

558 of tendons [J]. J Nanjing. University of Traditional Chinese Medicine;

$559 \quad 2014 ; 30: 14-6$.

560 6. Qiang X, Chaohui Z, Chaojun Z. Exploring the main points of repairing

561 the necrotic wound of diabetic foot tendon based on the phenomenon of

562 bloodletting of tendons. J Tianjin Univ Trad Chin Med. 2014;33:5-7. 
563 7. Bakker K, Apelqvist J, Schaper NC, International Working Group on

564 Diabetic Foot Editorial Board. Practical guidelines on the management and

565 prevention of the diabetic foot 2011. Diabetes Metab Res Rev. 2012;28:225-31.

566 8. Ying T, Chaohui Z, Jing M, Chaojun Z. Application of bromelain

567 combined with Shengji ointment in bed preparation of diabetic foot wound.

568 Chin J Trad Chin Med Inf. 2012;19:74-5.

569 9. Chaohui Z, Qiang X, Jing M, Xianzhou L, Chaojun Z. Experimental

570 study on the treatment of diabetic tendon necrosis model rats by chemical decay

571 and regeneration. Shi Zhen Guo Yi Guo Yao. 2011;22:223-6.

572 10. Qiang Zhang Chaohui X, Jing M, Chaojun Z, Xianzhou L. Preliminary

573 study on the treatment of diabetic foot by the method of blood and decay

574 regeneration of tendons. J Nanjing. University of Traditional Chinese Medicine.

$575 \quad 2014 ; 30: 14-6$.

576 11. Technical guidelines for clinical research of new Chinese medicines for

577 diabetic foot disease (draft for comments). Retrieved from:

578 http://www.cde.org.cn/news.do?method=largeInfo\&id=07274f255ba232d2

579 12. Xue YM, Zou MC. Interpretation of the Chinese guidelines for the

580 prevention and treatment of diabetic foot. 2019 ed. [J]. Chinese Journal of

581 Diabetes. 2019;2:88-91. 
582 13. Chinese guidelines for the prevention and treatment of diabetic foot. 2019

583 ed. II [J]. Chinese Journal of Diabetes. 2019;3:161-89.

584 14. Chinese guidelines for the prevention and treatment of diabetic foot. 2019

585 ed. III [J]. Chinese Journal of Diabetes. 2019;4:238-47.

586 15. Chinese guidelines for the prevention and treatment of diabetic foot. 2019

587 ed. IV [J]. Chinese Journal of Diabetes. 2019;5:316-27.

588 16. Chinese guidelines for the prevention and treatment of diabetic foot. 2019

589 ed. V [J]. Chinese Journal of Diabetes. 2019;6:387-97. 
Tables

591 Table 1 Study time-period

\begin{tabular}{|c|c|c|c|c|}
\hline & \multicolumn{4}{|c|}{ STUDY PERIOD } \\
\hline & Enrolment & Allocation & Post-allocation & Close-out \\
\hline TIMEPOINT & -1 day & 0 day & After 14 days & After 28 days \\
\hline \multicolumn{5}{|l|}{ ENROLLMENT: } \\
\hline \multirow{2}{*}{$\begin{array}{l}\text { Eligibility screen } \\
\text { Informed consent }\end{array}$} & - & & & \\
\hline & $\bullet$ & & & \\
\hline Allocation & & $\bullet$ & & \\
\hline \multicolumn{5}{|l|}{ INTERVENTIONS: } \\
\hline $\begin{array}{r}\text { Treatment group: Shengji ointment and } \\
\text { Pineapple protease (bromelain) }\end{array}$ & & $\bullet$ & $\bullet$ & $\bullet$ \\
\hline Control group: Comfeel® Plus wound & & $\bullet$ & $\bullet$ & $\bullet$ \\
\hline
\end{tabular}




\begin{tabular}{|c|c|c|c|c|}
\hline \multicolumn{5}{|l|}{ ASSESSMENTS: } \\
\hline Demographic data & $\bullet$ & & & \\
\hline Medical history and allergy history & $\bullet$ & & & \\
\hline Vital signs & & $\bullet$ & $\bullet$ & $\bullet$ \\
\hline Physical examination & & $\bullet$ & $\bullet$ & $\bullet$ \\
\hline Blood routine $+\mathrm{C}$ - reactive protein & & $\bullet$ & & $\bullet$ \\
\hline Urine routine & & $\bullet$ & & $\bullet$ \\
\hline Routine stool + occult blood & & $\bullet$ & & $\bullet$ \\
\hline Liver function & & $\bullet$ & & $\bullet$ \\
\hline Renal function & & $\bullet$ & & $\bullet$ \\
\hline Electrocardiogram & & $\bullet$ & & $\bullet$ \\
\hline Urine pregnancy test & & $\bullet$ & & \\
\hline
\end{tabular}




\begin{tabular}{|c|c|c|c|}
\hline Wound coverage rate & $\bullet$ & - & • \\
\hline Wound healing rate & - & - & - \\
\hline Amputation extent & & - & - \\
\hline Granulation time & & - & • \\
\hline Maryland Foot Score & • & $\bullet$ & • \\
\hline Clearance time & & - & • \\
\hline Granulation tissue score & - & & $\bullet$ \\
\hline Adverse events & - & - & $\bullet$ \\
\hline
\end{tabular}




\section{$594 \quad$ Additional files}

595 File name: Additional file 1

596 File format: .doc

597 Title of data: Standard operating procedure for wound photographing

598 Description of data: Additional file 1 describes the standard operating procedure

599 for taking pictures of the diabetic foot ulcers.

600

601 File name: Additional file 2

602 File format: .doc

603 Title of data: Standard operating procedure for wound debridement and dressing

604 change

605 Description of data: Additional file 2 describes the standard operating procedure

606 for the wound debridement and dressing changes.

607

608 File name: Additional file 3

609 File format: .doc 
610 Title of data: Maryland Foot Score evaluation standard

611 Description of data: Additional file 3 describes the Maryland Foot Score

612 evaluation standard. It is a secondary outcome used to evaluate the foot function.

613

614 File name: Additional file 4

615 File format: doc

616 Title of data: Scoring of granulation tissue growth in wounds

617 Description of data: Additional file 4 describes the scoring of granulation tissue

618 growth in wounds.

619

620 Additional file 5:

621 File name: Additional file 5

622 File format: .doc

623 Title of data: Ethical approval document

624 Description of data: Additional file 5 is the copy of the ethical approval

625 document and its English translation. 
626

627 File name: Additional file 6

628 File format: .doc

629 Title of data: Copy of the original funding documentation.

630 Description of data: Additional file 6 is the copy of the original funding

631 documentation and its English translation.

632 


\section{Supplementary Files}

This is a list of supplementary files associated with this preprint. Click to download.

- SPIRITChecklist.pdf

- Additionalfile1.pdf

- Additionalfile2.pdf

- Additionalfile3.pdf

- Additionalfile4.pdf 